Homology, Homotopy and Applications, vol.10(1), 2008, pp.205-222

\title{
BETTI NUMBERS OF RANDOM MANIFOLDS
}

\author{
MICHAEL FARBER AND THOMAS KAPPELER
}

\author{
(communicated by Robert Ghrist)
}

\begin{abstract}
We study mathematical expectations of Betti numbers of configuration spaces of planar linkages, viewing the lengths of the bars of the linkage as random variables. Our main result gives an explicit asymptotic formulae for these mathematical expectations for two distinct probability measures describing the statistics of the length vectors when the number of links tends to infinity. In the proof we use a combination of geometric and analytic tools. The average Betti numbers are expressed in terms of volumes of intersections of a simplex with certain half-spaces.
\end{abstract}

\section{Introduction}

In various fields of applications, such as topological robotics, configuration spaces of mechanical systems depend on a large number of parameters, which typically are only partially known and often can be considered as random variables. Since these parameters determine the topology of the configuration space, the latter can be viewed in such a case as a random topological space or a random manifold. To control such a system one has to understand geometry, topology and control theory of random manifolds.

One of the most natural notions to investigate is the mathematical expectation of the Betti numbers of random manifolds. Clearly, these average Betti numbers encode valuable information for engineering applications; for instance they provide an average lower bound for the number of critical points of a Morse function (i.e. observable) on such manifolds.

In this paper we consider a specific instance of this general problem. We study closed planar $n$-gons whose sides have fixed positive lengths $l_{1}, \ldots, l_{n}$. The "polygon" space

$$
M_{\ell}=\left\{\left(u_{1}, \ldots, u_{n}\right) \in S^{1} \times \cdots \times S^{1} ; \sum_{i=1}^{n} l_{i} u_{i}=0 \in \mathbf{C}\right\} / \operatorname{SO}(2)
$$

parametrizes the variety of all possible shapes of such planar $n$-gons with sides of length $l_{1}, \ldots, l_{n}$. The unit vector $u_{i} \in \mathbf{C}$ indicates the direction of the $i$-th side of the

Received June 5, 2007; published on February 22, 2008.

2000 Mathematics Subject Classification: 55R 80.

Key words and phrases: linkage, polygon space, random linkage, Betti number.

This article is available at http://intlpress.com/HHA/v10/n1/a8

Copyright (c) 2008, Michael Farber and Thomas Kappeler. Permission to copy for private use granted. 
polygon. The condition $\sum l_{i} u_{i}=0$ expresses the property of the polygon being closed. The rotation group $\mathrm{SO}(2)$ acts on the set of side directions $\left(u_{1}, \ldots, u_{n}\right)$ diagonally.

The polygon space $M_{\ell}$ emerges in topological robotics as the configuration space of the planar linkage, a simple mechanism consisting of $n$ bars of length $l_{1}, \ldots, l_{n}$ connected by revolving joints forming a closed planar polygonal chain. The positions of two adjacent vertices are fixed but the other vertices are free to move and the angles between the bars are allowed to change. The spaces $M_{\ell}$ also appear in molecular

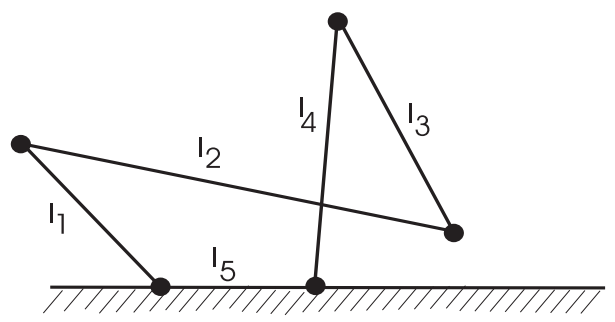

biology, where they describe the space of shapes of closed molecular chains.

Statistical shape theory is another subject where the spaces $M_{\ell}$ play a role: they describe the space of shapes having certain geometric properties with respect to the central point; see $[\mathbf{8}]$.

The configuration space $M_{\ell}$ depends on the length vector

$$
\ell=\left(l_{1}, \ldots, l_{n}\right) \in \mathbf{R}_{+}^{n}
$$

in an essential way. Here $\mathbf{R}_{+}^{n}$ denotes the set of vectors in $\mathbf{R}^{n}$ having nonnegative coordinates. Clearly, $M_{\ell}=M_{t \ell}$ for any $t>0$.

The length vector $\ell$ is called generic if $\sum_{i=1}^{n} l_{i} \epsilon_{i} \neq 0$ for any choice $\epsilon_{i}= \pm 1$. It is known that for a generic length vector $\ell$ the space $M_{\ell}$ is a closed smooth manifold of dimension $n-3$. If the length vector $\ell$ is not generic, then $M_{\ell}$ is a compact $(n-3)$ dimensional manifold having finitely many singular points.

The moduli spaces $M_{\ell}$ of planar polygonal linkages were studied extensively by W. Thurston and J. Weeks [11], K. Walker [13], A. A. Klyachko [9], M. Kapovich and J. Millson [7], J.-Cl. Hausmann and A. Knutson [6] and others. The Betti numbers of $M_{\ell}$ as functions of the length vector $\ell$ are described in [4]; we recall the result of [4] later in Section 2. A. A. Klyachko [9] found the Betti numbers of spatial polygon spaces.

In this paper we view the length vector $\ell \in \mathbf{R}_{+}^{n}$ as a random variable whose statistical behavior is described by a probability measure $\mu$ on $\mathbf{R}_{+}^{n}$. The $p$-dimensional Betti number $b_{p}\left(M_{\ell}\right)$ is then a function

$$
b_{p}: \mathbf{R}_{+}^{n} \rightarrow \mathbf{Z}, \quad \ell \mapsto b_{p}\left(M_{\ell}\right) \in \mathbf{Z} .
$$

Our goal is to find the asymptotics for $n$ large of the average Betti numbers, defined by

$$
b_{p}(n, \mu)=\int_{\mathbf{R}_{+}^{n}} b_{p}\left(M_{\ell}\right) d \mu .
$$


The choice of measure $\mu$ reflects a priori information about the problem. We study in detail two special choices for $\mu$ :

(a) $\mu=\mu_{a}$ is the probability measure on $\mathbf{R}_{+}^{n}$ supported on the unit simplex $\Delta^{n-1}$ such that $\left.\mu\right|_{\Delta^{n-1}}$ coincides with the Lebesgue measure on $\Delta^{n-1} \subset \mathbf{R}_{+}^{n}$ normalized so that $\mu\left(\Delta^{n-1}\right)=1$. Recall that $\Delta^{n-1} \subset \mathbf{R}_{+}^{n}$ is described by the inequalities $l_{i} \geqslant 0, \sum l_{i}=1$.

(b) $\mu=\mu_{b}$ is the probability measure on $\mathbf{R}_{+}^{n}$ supported on the unit cube $\square^{n} \subset \mathbf{R}_{+}^{n}$ such that $\left.\mu\right|_{\square^{n}}$ is the Lebesgue measure. Here $\square^{n}$ is given by the inequalities $0 \leqslant l_{i} \leqslant 1, i=1, \ldots, n$ and $\mu\left(\square^{n}\right)=1$.

The main result of this paper states that in cases (a) and (b) for any fixed $p \geqslant 0$ and large $n$ the following asymptotic formula holds:

$$
b_{p}(n, \mu) \sim\left(\begin{array}{c}
n-1 \\
p
\end{array}\right) .
$$

This can be expressed by saying that for large $n$ the random manifold $M_{\ell}$ is connected, its first Betti number is $n-1$, the second is $(n-1)(n-2) / 2$ and so on. More precisely, our main result is the following:

Theorem 1.1. Let $\mu$ be either of the measures $\mu_{a}$ or $\mu_{b}$ described above. Then for any $p \geqslant 0$ there exist constants $C>0$ and $0<a<1$ such that for any $n$ the average Betti number $b_{p}(n, \mu)$, given by (4), satisfies

$$
\left|b_{p}(n, \mu)-\left(\begin{array}{c}
n-1 \\
p
\end{array}\right)\right|<C a^{n} .
$$

This result is quite surprising for two reasons. Firstly, it states that the asymptotic values of the average Betti number $b_{p}(n, \mu)$ as $n \rightarrow \infty$ are equal for the measures $\mu_{a}$ and $\mu_{b}$ and raises the intriguing question about the universality of the obtained asymptotic values.

Secondly, the binomial coefficient which appears in estimate (6) equals the $p$ dimensional Betti number $b_{p}\left(M_{\ell^{*}}\right)$ of the configuration space of the equilateral linkage $\ell^{*}=(1, \ldots, 1)$ for any $p$ satisfying $2 p<n-3$; see [4, Examples 3 and 4]. Hence the Betti numbers of the moduli space $M_{\ell^{*}}$ approximate the average Betti numbers $b_{p}(n, \mu)$ for $n$ large. On the other hand, by Theorem 2 of [4] the total Betti number, $\sum_{p=0}^{n} b_{p}\left(M_{\ell}\right)$, viewed as a function of the length vector $\ell \in \mathbf{R}_{+}^{n}$, is maximal for the equilateral linkage $\ell=\ell^{*}$. This comparison shows that at least for some values of $p$ the maximum of the individual Betti numbers $b_{p}\left(M_{\ell}\right)$ viewed as a function of $\ell$ must be higher than $b_{p}\left(M_{\ell^{*}}\right)$.

Indeed, this is the case since for the length vector $\ell_{\epsilon}=(1,1,1, \epsilon, \ldots, \epsilon)$, where $\epsilon$ is a small positive number which appears $n-3$ times, the Betti number $b_{p}\left(M_{\ell_{\epsilon}}\right)$ equals

$$
2 \cdot\left(\begin{array}{c}
n-3 \\
p
\end{array}\right)=2 \cdot \frac{(n-2-p)(n-1-p)}{(n-1)(n-2)} \cdot\left(\begin{array}{c}
n-1 \\
p
\end{array}\right)
$$

(see [4, Example 2]), which for large $n$ is nearly twice the average Betti number 
$b_{p}(n, \mu)$. However if $p \sim n / 2 \mathrm{x}$, then

$$
b_{p}\left(M_{\ell_{\epsilon}}\right) \sim \frac{1}{2}\left(\begin{array}{c}
n-1 \\
p
\end{array}\right) .
$$

In a subsequent work we shall describe a generalization of Theorem 1.1 which allows the dimension $p$ to grow with $n$. See also [3] where the average Betti numbers of polygon spaces in $\mathbf{R}^{3}$ are calculated. Paper [3] also contains results for more general probability measures which explain the "universality phenomenon".

\section{Reduction of the problem}

In this section we express the average Betti numbers in terms of certain volumes.

To state this result we need to introduce some more notation. For a subset $J \subset\{1, \ldots, n\}$ we denote by $\phi_{J}: \mathbf{R}^{n} \rightarrow \mathbf{R}$ the linear functional given by

$$
\phi_{J}\left(l_{1}, \ldots, l_{n}\right)=\sum_{i \in J} l_{i}-\sum_{i \notin J} l_{i}
$$

and by $H_{J}$ the half-space

$$
H_{J}=\left\{\ell \in \mathbf{R}^{n} ; \phi_{J}(\ell)<0\right\} .
$$

Further, $C^{n}$ denotes the cone

$$
C^{n}=\left\{\left(l_{1}, \ldots, l_{n}\right) ; l_{1} \geqslant l_{2} \geqslant \ldots l_{n} \geqslant 0\right\} \subset \mathbf{R}_{+}^{n} .
$$

The main result of this section provides a reduction of the problem of computing the average Betti numbers.

Proposition 2.1. Let $\mu$ be a probability measure on $\mathbf{R}_{+}^{n}$ having the following two properties:

(I) $\mu$ is invariant with respect to the action of the symmetric group $\Sigma_{n}$ on $\mathbf{R}_{+}^{n}$ permuting coordinates;

(II) $\mu\left(L \cap \mathbf{R}_{+}^{n}\right)=0$ for any proper linear subspace $L \subset \mathbf{R}^{n}$.

Then the average Betti number $b_{p}(n, \mu)$ equals

$$
b_{p}(n, \mu)=n ! \cdot \sum_{J} \mu\left(H_{J} \cap C^{n}\right),
$$

where $J$ runs over all subsets $J \subset\{1,2, \ldots, n\}$ containing 1 and having cardinality either $|J|=p+1$ or $|J|=n-2-p$.

Proof. First, recall the result of $[4]$ stating that the Betti numbers $b_{p}\left(M_{\ell}\right)$, as functions of the length vector $\ell=\left(l_{1}, \ldots, l_{n}\right)$, can be computed by counting certain subsets of the index set $\{1, \ldots, n\}$. A subset $J \subset\{1, \ldots, n\}$ is called short if

$$
\sum_{i \in J} l_{i}<\sum_{i \notin J} l_{i} .
$$

A subset is called long if its complement is short. A subset $J \subset\{1, \ldots, n\}$ is called 
median if

$$
\sum_{i \in J} l_{i}=\sum_{i \notin J} l_{i}
$$

Fix an index $1 \leqslant i \leqslant n$ such that $l_{i}$ is maximal among $l_{1}, \ldots, l_{n}$. Denote by $a_{p}(\ell)$ the number of short subsets $J \subset\{1, \ldots, n\}$ of cardinality $|J|=1+p$ containing $i$. Denote by $\tilde{a}_{p}(\ell)$ the number of median subsets $J \subset\{1, \ldots, n\}$ containing $i$ and such that $|J|=1+p$. It was proven in $[4]$ that for $p=0,1, \ldots, n-3$ one has

$$
b_{p}\left(M_{\ell}\right)=a_{p}(\ell)+\tilde{a}_{p}(\ell)+a_{n-3-p}(\ell) .
$$

It is easy to see that the manifolds $M_{\ell_{1}}$ and $M_{\ell_{2}}$ are diffeomorphic if the length vector $\ell_{1} \in \mathbf{R}_{+}^{n}$ is obtained from $\ell_{2} \in \mathbf{R}_{+}^{n}$ by permuting the components. In other words, for our purposes, the order of the coordinates $l_{1}, \ldots, l_{n}$ in the length vector $\ell=\left(l_{1}, \ldots, l_{n}\right)$ is irrelevant.

Let $\mu$ be a probability measure on $\mathbf{R}_{+}^{n}$ having properties (I) and (II). Property (II) implies that

$$
\int_{\mathbf{R}_{+}^{n}} \tilde{a}_{p}(\ell) d \mu=0
$$

since the function $\ell \mapsto \tilde{a}_{p}(\ell)$ is zero on the complement of a union of finitely many linear hyperplanes. Integrating (11) we have

$$
b_{p}(n, \mu)=a_{p}(n, \mu)+a_{n-3-p}(n, \mu),
$$

where

$$
a_{p}(n, \mu)=\int_{\mathbf{R}_{+}^{n}} a_{p}(\ell) d \mu=n ! \cdot \int_{C^{n}} a_{p}(\ell) d \mu .
$$

The latter identity follows from property (I) of $\mu$. The function $\left.a_{p}\right|_{C^{n}}$ is quite simple. Denote by $\sigma$ the step function

$$
\sigma(x)= \begin{cases}1, & \text { if } x<0 \\ 0, & \text { if } x \geqslant 0\end{cases}
$$

where $x \in \mathbf{R}$. Then we may write

$$
a_{p}(\ell)=\sum_{J} \sigma\left(\left(\phi_{J}(\ell)\right)\right.
$$

where $J$ runs over all subsets of $\{1, \ldots, n\}$ of cardinality $p+1$ containing 1 . Integrating we find

$$
\begin{aligned}
a_{p}(n, \mu) & =n ! \cdot \int_{C^{n}} a_{p}(\ell) d \mu \\
& =n ! \cdot \sum_{J} \int_{C^{n}} \sigma\left(\phi_{J}(\ell)\right) d \mu .
\end{aligned}
$$

In formula (16), $J$ runs over all subsets $J \subset\{1, \ldots, n\}$ satisfying $1 \in J,|J|=p+1$. 
Obviously,

$$
\int_{C^{n}} \sigma\left(\phi_{J}(\ell)\right) d \mu=\mu\left(H_{J} \cap C^{n}\right),
$$

where $H_{J}$ is given by (8). Our statement (10) now follows by combining (13), (14), (16) and (17).

\section{Simplices and volumes}

In this section we give a geometric interpretation of the quantities appearing on the right-hand side of formula (10).

Recall that we denote by $\mu_{a}$ the measure on $\mathbf{R}_{+}^{n}$ with support on the unit simplex $\Delta^{n-1} \subset \mathbf{R}_{+}^{n}$ such that $\left.\mu_{a}\right|_{\Delta^{n-1}}$ coincides with the normalized Lebesgue measure, $\mu_{a}\left(\Delta^{n-1}\right)=1$. Similarly, $\mu_{b}$ denotes the probability measure on $\mathbf{R}_{+}^{n}$ supported on the unit cube $\square^{n} \subset \mathbf{R}_{+}^{n}$ such that $\left.\mu_{b}\right|_{\square^{n}}$ coincides with the Lebesgue measure.

Denote by $A \subset \mathbf{R}_{+}^{n}$ the simplex of dimension $n$ having the vertices

$$
\begin{aligned}
c_{0}= & (0, \ldots, 0), \\
c_{1}= & (1,0, \ldots, 0), \\
c_{2}= & \frac{1}{2}(1,1,0, \ldots, 0), \\
& \ldots \\
c_{n}= & \frac{1}{n}(1,1, \ldots, 1) .
\end{aligned}
$$

Similarly, denote by $B \subset \mathbf{R}_{+}^{n}$ the simplex of dimension $n$ having the vertices

$$
c_{i}^{\prime}=(\underbrace{1, \ldots, 1}_{i \text { times }}, 0, \ldots, 0)=i \cdot c_{i}
$$

where $i=0,1, \ldots, n$.

Proposition 3.1. For any subset $J \subset\{1, \ldots, n\}$, one has

$$
n ! \cdot \mu_{a}\left(H_{J} \cap C^{n}\right)=\frac{\operatorname{vol}\left(H_{J} \cap A\right)}{\operatorname{vol}(A)}, \quad n ! \cdot \mu_{b}\left(H_{J} \cap C^{n}\right)=\frac{\operatorname{vol}\left(H_{J} \cap B\right)}{\operatorname{vol}(B)} .
$$

In other words, the quantities appearing in Proposition 2.1 can be interpreted as ratios of volumes of certain simplices and the parts cut off by a half-space.

Proof. One checks that the intersection $\Delta^{n-1} \cap C^{n}$ coincides with the $(n-1)$-dimensional simplex $A^{\prime} \subset A$ with vertices $c_{1}, \ldots, c_{n}$, and that the intersection $\square^{n} \cap C^{n}$ equals $B$. Clearly

$$
\mu_{a}\left(A^{\prime}\right)=\frac{\operatorname{vol}_{n-1}\left(A^{\prime}\right)}{\operatorname{vol}_{n-1}(A)}=(n !)^{-1}, \quad \mu_{b}(B)=\operatorname{vol}(B)=(n !)^{-1} .
$$

Here $\operatorname{vol}_{n-1}$ denotes the $(n-1)$-dimensional Euclidean volume. Hence we find that

$$
n ! \cdot \mu_{a}\left(H_{J} \cap C^{n}\right)=\frac{\operatorname{vol}_{n-1}\left(H_{J} \cap A^{\prime}\right)}{\operatorname{vol}_{n-1}\left(A^{\prime}\right)}=\frac{\operatorname{vol}\left(H_{J} \cap A\right)}{\operatorname{vol}(A)}
$$


and

$$
n ! \cdot \mu_{b}\left(H_{J} \cap C^{n}\right)=\frac{\operatorname{vol}\left(H_{J} \cap B\right)}{\operatorname{vol}(B)} .
$$

This completes the proof.

We are led to consider the following simple geometric problem.

Proposition 3.2. Given $n+1$ points $v_{0}, \ldots, v_{n} \in \mathbf{R}^{n}$ in general position and a linear functional $\phi: \mathbf{R}^{n} \rightarrow \mathbf{R}$, consider the simplex $\Sigma$ spanned by $v_{0}, \ldots, v_{n}$ and its intersection with the half-space $H=\left\{v \in \mathbf{R}^{n} ; \phi(v)<0\right\}$. Denote by

$$
q_{i}=\phi\left(v_{i}\right), \quad i=0, \ldots, n
$$

the values of the functional $\phi$ on the vertices of $\Sigma$. Assume that the numbers $q_{0}, \ldots, q_{n}$ are all distinct and that the vertices are labelled so that $q_{i}<0$ for all $i=0, \ldots, m$ and $q_{i} \geqslant 0$ for $i=m+1, \ldots, n$. Then the ratio of the volumes

$$
r=\frac{\operatorname{vol}(\Sigma \cap H)}{\operatorname{vol}(\Sigma)}
$$

equals

$$
r=\sum_{i=0}^{m} \prod_{\substack{0 \leqslant n \\ j \neq i}} \frac{q_{i}}{q_{i}-q_{j}}
$$

Proposition 3.2 is well known. Different expressions for the volume cut off of a simplex by a half-space were obtained in [2] (see Theorem 2 of [2]) and more recently in $[\mathbf{1}],[\mathbf{5}],[\mathbf{1 2}]$. Proposition 3.2 can be easily obtained from the results mentioned above. For the convenience of the reader we briefly sketch the proof.

Proof. Consider for $x \in \mathbf{R}$ the half-space

$$
H_{x}=\left\{v \in \mathbf{R}^{n} ; \phi(v)<x\right\}
$$

and the real-valued function

$$
r(x)=\frac{\operatorname{vol}\left(H_{x} \cap B\right)}{\operatorname{vol}(B)} .
$$

Without loss of generality we will assume that $q_{0}<q_{1}<\cdots<q_{n}$; for convenience we set $q_{-1}=-\infty$ and $q_{n+1}=+\infty$.

The function $r(x)$ has the following properties:

(i) when restricted on each subinterval $\left[q_{i-1}, q_{i}\right]$, the function $r(x)$ is a polynomial $p_{i}(x)$ of degree $n$ with real coefficients, where $i=0, \ldots, n+1$.

(ii) $p_{0}(x) \equiv 0$ and $p_{n+1}(x) \equiv 1$.

(iii) $r(x)$ has continuous derivatives up to order $n-1$. 
Properties (i) and (iii) (proven in [2]) imply that for any $0 \leqslant i \leqslant n$ there exists $\beta_{i} \in \mathbf{R}$ such that

$$
p_{i+1}(x)-p_{i}(x)=\beta_{i}\left(x-q_{i}\right)^{n} .
$$

Hence

$$
p_{i+1}(x)=\sum_{k=0}^{i} \beta_{k}\left(x-q_{k}\right)^{n},
$$

where the coefficients $\beta_{i}$ can be found from the polynomial identity

$$
\sum_{k=0}^{n} \beta_{k}\left(x-q_{k}\right)^{n} \equiv 1 .
$$

Equation (29) has a unique solution given by

$$
\beta_{k}=(-1)^{n} \cdot \prod_{\substack{j=0 \\ j \neq k}}^{n}\left(q_{k}-q_{j}\right)^{-1}, \quad k=0,1, \ldots, n .
$$

Indeed, by comparison of coefficients in (29), we obtain the linear system

$$
\sum_{k=0}^{n} \beta_{k} q_{k}^{n-i}= \begin{cases}(-1)^{n} & \text { if } i=0, \\ 0 & \text { if } i=1, \ldots, n,\end{cases}
$$

which can be written in the matrix form

$$
\left[\begin{array}{cccc}
1 & 1 & \ldots & 1 \\
q_{0} & q_{1} & \ldots & q_{n} \\
\ldots & \ldots & \ldots & \ldots \\
q_{0}^{n-1} & q_{1}^{n-1} & \ldots & q_{n}^{n-1} \\
q_{0}^{n} & q_{1}^{n} & \ldots & q_{n}^{n}
\end{array}\right]\left[\begin{array}{c}
\beta_{0} \\
\beta_{1} \\
\ldots \\
\beta_{n-1} \\
\beta_{n}
\end{array}\right]=\left[\begin{array}{c}
0 \\
0 \\
\ldots \\
0 \\
(-1)^{n}
\end{array}\right]
$$

To solve it one applies Cramer's rule. The determinant of the system is the Vandermonde determinant $\prod_{j>l}\left(q_{j}-q_{l}\right)$, and the numerator of the fraction expressing $\beta_{k}$ can be computed to be the Vandermonde determinant

$$
(-1)^{k} \cdot \prod_{\substack{j>l \\ j, l \neq k}}\left(q_{j}-q_{l}\right),
$$

so that we obtain

$$
\beta_{k}=(-1)^{k} \prod_{k>l}\left(q_{k}-q_{l}\right)^{-1} \cdot \prod_{j>k}\left(q_{j}-q_{k}\right)^{-1}=(-1)^{n} \prod_{j \neq k}\left(q_{k}-q_{j}\right)^{-1},
$$

which coincides with (30). Substituting the obtained value into (28) we find that for 
$x \in\left[q_{i-1}, q_{i}\right]$ one has

$$
r(x)=p_{i}(x)=\sum_{k=0}^{i-1} \prod_{\substack{0 \leqslant j \leqslant n \\ j \neq k}} \frac{q_{k}-x}{q_{k}-q_{j}} .
$$

Formula (25) is obtained from (33) by setting $x=0$.

For our applications we need to have a more general formula for the ratio (24) which covers the case when some values $q_{i}=\phi\left(c_{i}\right)$ coincide. Formula (25) is not well defined in this case since some of the denominators might vanish. Our setting is as follows. Let $v_{0}, \ldots, v_{n} \in \mathbf{R}^{n}$ be points in general position spanning a simplex $B$. Let $\phi: \mathbf{R}^{n} \rightarrow \mathbf{R}$ be a linear functional. We consider the ratio of the volumes (25) where $H$ is the half-space $H=\left\{v \in \mathbf{R}^{n} ; \phi(v)<0\right\}$. Let $q_{i}=\phi\left(v_{i}\right) \in \mathbf{R}$, where $i=0, \ldots, n$. Suppose that there is a decomposition of the set of indices into disjoint subsets

$$
\{0,1, \ldots, n\}=\bigsqcup_{l=0}^{s} I_{l}
$$

such that $q_{i}=Q_{l}$ for all $i \in I_{l}$ and the numbers $Q_{0}, \ldots, Q_{s} \in \mathbf{R}$ are pairwise distinct. We denote by $k_{l}$ the number $\left|I_{l}\right|-1$. Thus, the multiplicity of $Q_{l}$ is $k_{l}+1$, where $l=0,1, \ldots, s$, and one has

$$
k_{0}+k_{1}+\cdots+k_{s}=n-s .
$$

Given two nonnegative integers $s$ and $a$, we denote by $P(s, a)$ the set of all functions

$$
\delta:\{0,1, \ldots, s\} \rightarrow \mathbf{Z}_{\geqslant 0}, \quad i \mapsto \delta_{i}
$$

satisfying $\sum_{j=0}^{s} \delta_{j}=a$. The set $P(s, a)$ labels partitions of $a$ in $s+1$ summands.

Proposition 3.3. Assume that $Q_{i}<0$ for all $i=0, \ldots, m$ and $Q_{i} \geqslant 0$ for all $i=$ $m+1, \ldots, s$. Then the ratio of volumes (24) equals

$$
r=\sum_{i=0}^{m}\left[F_{i} \cdot \prod_{\substack{j \leqslant s \\ j \neq i}}\left(\frac{Q_{i}}{Q_{i}-Q_{j}}\right)^{k_{j}+1}\right]
$$

where $F_{i}$ equals

$$
\sum_{\delta \in P\left(s, k_{i}\right)}\left(\begin{array}{c}
n \\
\delta_{i}
\end{array}\right) \cdot\left(-Q_{i}\right)^{k_{i}-\delta_{i}} \cdot \prod_{\substack{0 \leqslant j \leqslant s \\
j \neq i}}\left(\begin{array}{c}
k_{j}+\delta_{j} \\
\delta_{j}
\end{array}\right) \cdot\left(Q_{i}-Q_{j}\right)^{-\delta_{j}}
$$

Note that if $k_{i}=0$, then $P\left(s, k_{i}\right)=\{\delta \equiv 0\}$, and hence $F_{i}=1$. In particular, if $s=n$ and therefore $k_{i}=0$ for any $0 \leqslant i \leqslant n$, then formula (36) coincides with formula (25). Moreover, we point out that the coefficients $F_{i}$ are homogenous of degree 0 in the variables $Q_{0}, \ldots, Q_{s}$. 
Proof of Proposition 3.3. Let $\psi: \mathbf{R}^{n} \rightarrow \mathbf{R}$ be a linear functional, a small perturbation of $\phi: \mathbf{R}^{n} \rightarrow \mathbf{R}$, such that the values $p_{i}=\psi\left(v_{i}\right)$ are all distinct. Without loss of generality we may assume that $p_{i}<0$ for $0 \leqslant i \leqslant m^{\prime}$ and $p_{i}>0$ for $m^{\prime}<i \leqslant n$, where $m^{\prime}=m+1+\sum_{i=0}^{m} k_{i}$. Let $H^{\prime}=\left\{v \in \mathbf{R}^{n} ; \psi(v)<0\right\}$. The perturbed ratio

$$
r^{\prime}=\frac{\operatorname{vol}\left(H^{\prime} \cap \Sigma\right)}{\operatorname{vol}(\Sigma)}
$$

tends to $r$ when $\psi$ tends to $\phi$. By Proposition 3.2 it can be written in the form

$$
r^{\prime}=\sum_{i=0}^{m^{\prime}}\left[p_{i}^{n} . \prod_{\substack{j \neq i \\ 0 \leqslant j \leqslant n}}\left(p_{i}-p_{j}\right)^{-1}\right] .
$$

To find its limit we will use the following general formula (see $[\mathbf{1 0}]$ ),

$$
\sum_{i=0}^{k} f\left(x_{i}\right) \cdot \prod_{\substack{0 \leqslant j \leqslant k \\ j \neq i}}\left(x_{i}-x_{j}\right)^{-1}=\frac{1}{k !} f^{(k)}(\xi),
$$

where $f(x)$ is a real-valued smooth function, $x_{0}, \ldots, x_{k}$ are distinct real numbers and $\xi$ is a number lying in the smallest interval containing these points. Applying (39) $(m+1)$ times to $(38)$ and passing to the limit as $\left\{p_{0}, \ldots, p_{n}\right\} \rightarrow\left\{Q_{0}, \ldots, Q_{s}\right\}$ we obtain

$$
r=\sum_{i=0}^{m} \frac{1}{k_{i} !}\left[x^{n} \cdot \prod_{\substack{k \leqslant s \\ l \neq i}}\left(x-Q_{l}\right)^{-k_{l}-1}\right]_{x=Q_{i}}^{\left(k_{i}\right)}
$$

Recall the following formula for higher derivatives of products

$$
\left(f_{0} f_{1} \cdots f_{s}\right)^{(k)}=\sum_{\delta \in P(s, k)} \frac{k !}{\delta_{0} ! \cdots \delta_{s} !} f_{0}^{\left(\delta_{0}\right)} \cdots f_{s}^{\left(\delta_{s}\right)} .
$$

Applying it to (40) and using $n=k_{i}+\sum_{j \neq i}\left(k_{j}+1\right)$, one obtains (35) after certain elementary transformations.

\section{Sequences of densities}

By Proposition 2.1 combined with Proposition 3.1, to compute the average Betti numbers $b_{p}(n, \mu)$ one has to know the volumes cut off of a simplex by certain halfspaces. The result of Propositions 3.2 and 3.3 show that to find these volumes it is enough to know the values of the functionals determining the half-spaces on the vertices of the simplices $A$ and $B$. We investigate these values in this section. 
Let $J \subset\{1, \ldots, n\}$ be an arbitrary subset and $\phi_{J}: \mathbf{R}^{n} \rightarrow \mathbf{R}$ be the linear functional (7). The values of the functional $\phi_{J}$ on the vertices $c_{i}$ of the simplex $A$ (see (18)) equal $\phi_{J}\left(c_{0}\right)=0$, and for $i \geqslant 1$,

$$
\phi_{J}\left(c_{i}\right)=\frac{1}{i}|J \cap\{1, \ldots, i\}|-\frac{1}{i}|\bar{J} \cap\{1, \ldots, i\}|,
$$

where $\bar{J}$ denotes the complement of $J$ in $\{1, \ldots, n\}$. Let

$$
\alpha_{i}(J)=\frac{1}{i}|J \cap\{1, \ldots, i\}|, \quad i=1, \ldots, n
$$

denote the density of the set $J$ in the interval $\{1, \ldots, i\}$. Clearly $0 \leqslant \alpha_{i}(J) \leqslant 1$.

Lemma 4.1. Let $J \subset\{1, \ldots, n\}$ be a subset of cardinality $|J|=p \geqslant 1$. Then the following estimates hold:

(a) For $2 p \leqslant i \leqslant n$ one has

$$
\alpha_{i}(J) \leqslant \frac{1}{2}
$$

and equality in (43) may only hold for $i=2 p$.

(b) If $1 \leqslant i, j \leqslant 2 p$, then either $\alpha_{i}(J)=\alpha_{j}(J)$ or

$$
\left|\alpha_{i}(J)-\alpha_{j}(J)\right| \geqslant \frac{1}{(2 p)^{2}} .
$$

(c) If $\alpha_{i}(J)<\frac{1}{2}$ for some $1 \leqslant i \leqslant n$, then

$$
\alpha_{i}(J) \leqslant \frac{1}{2}-\frac{1}{2(2 p+1)} .
$$

(d) For any $8 p^{3} \leqslant i \leqslant n$ one has

$$
0 \leqslant \alpha_{i}(J) \leqslant \frac{1}{8 p^{2}}
$$

Proof.

(a) Let $k_{i}$ denote $|J \cap\{1,2, \ldots, i\}|$. Then for $i \geqslant 2 p$ one has

$$
\alpha_{i}(J)=\frac{k_{i}}{i} \leqslant \frac{p}{i} \leqslant \frac{p}{2 p}=1 / 2 .
$$

If $\alpha_{i}(J)=1 / 2$, then the above inequalities imply that $i=2 p$.

(b) Suppose now that $1 \leqslant i, j \leqslant 2 p$. Then

$$
\left|\alpha_{i}(J)-\alpha_{j}(J)\right|=\left|\frac{k_{i}}{i}-\frac{k_{j}}{j}\right|=\frac{\left|j k_{i}-i k_{j}\right|}{i j} .
$$

We obtain that either (45) vanishes or $\left|j k_{i}-i k_{j}\right| \geqslant 1$; hence (45) is greater than or equal to

$$
\frac{1}{i j} \geqslant \frac{1}{(2 p)^{2}}
$$

proving (b). 
(c) Note that for $i \geqslant 2 p+2$ one has

$$
\alpha_{i}(J)=\frac{k_{i}}{i} \leqslant \frac{p}{2 p+2}=\frac{1}{2}-\frac{1}{2(p+1)} .
$$

Now consider the case $i \leqslant 2 p+1$. By assumption $\alpha_{i}(J)<1 / 2$, i.e. $2 k_{i}<i$ or $2 k_{i} \leqslant i-1$. It implies that

$$
\alpha_{i}(J)=\frac{k_{i}}{i} \leqslant \frac{i-1}{2 i}=\frac{1}{2}-\frac{1}{2 i} \leqslant \frac{1}{2}-\frac{1}{2(2 p+1)} .
$$

(d) Note that if $i \geqslant 8 p^{3}$ and $p \geqslant 1$, then

$$
\alpha_{i}(J)=\frac{k_{i}}{i} \leqslant \frac{p}{i} \leqslant \frac{1}{8 p^{2}} .
$$

Lemma 4.2. Let $J \subset\{1, \ldots, n\}$ with $|J|=p \geqslant 1$. A nonzero number may appear in the sequence of densities $\alpha_{1}(J), \ldots, \alpha_{n}(J)$ at most $p$ times.

Proof. The densities $\alpha_{i}(J)$ satisfy the following recurrence relation:

$$
\alpha_{i+1}(J)= \begin{cases}\frac{i}{i+1} \alpha_{i}(J), & \text { if } i+1 \notin J, \\ \frac{i}{i+1} \alpha_{i}(J)+\frac{1}{i+1}, & \text { if } i+1 \in J .\end{cases}
$$

It follows that $\alpha_{i+1}(J)<\alpha_{i}(J)$ if $\alpha_{i}(J)>0$ and $i+1 \notin J$. On the other hand, one has $\alpha_{i+1}(J) \geqslant \alpha_{i}(J)$ if $i+1 \in J$. Hence, for $i<j$, the equality $\alpha_{i}(J)=\alpha_{j}(J)>0$ implies that at least one of the intermediate indices $i+1, \ldots, j$ belongs to $J$.

Assume now that for $i_{1}<i_{2}<\cdots<i_{k}$ one has $\alpha_{i_{1}}(J)=\cdots=\alpha_{i_{k}}(J)>0$. The set $J$ divides $\{1, \ldots, n\}$ into $p+1$ subintervals, i.e. subsets of consecutive integers in $\{1, \ldots, n\}$ all of which are not in $J$ except the first one. The leftmost interval contains no elements of $J$ and might be empty. As explained above, each of the subintervals may contain at most one of the integers $i_{1}, \ldots, i_{k}$. The density $\alpha_{i}(J)$ vanishes if and only if $i$ lies in the leftmost subinterval. This shows that $k \leqslant p$ as claimed.

The following examples show that Lemma 4.2 cannot be improved.

\section{Example 4.3.}

(i) Consider $J=\{1,2, \ldots, p\}$. Then $\alpha_{i}(J)=1$ if and only if $1 \leqslant i \leqslant p$; i.e. the multiplicity of the value 1 is $p$.

(ii) Let $J=\{1,3,5, \ldots, 2 p-1\}$. Then $\alpha_{i}(J)$ equals $1 / 2$ exactly $p$ times.

(iii) Suppose that $J=\{n-p+1, n-p+2, \ldots, n\}$. Then one has $\alpha_{i}(J)=0$ for $i=$ $1,2, \ldots, n-p$; i.e. the multiplicity of the value zero is $n-p$. This shows that the bound of the multiplicity of Lemma 4.2 does not hold for the value zero.

The values

$$
q_{i}=\phi_{J}\left(c_{i}\right)
$$

of the functional $\phi_{J}$ on the vertices of the simplex $A$ (see (18)) will play an important role in the sequel. Clearly, $q_{0}=0$ and

$$
q_{i}=2 \alpha_{i}(J)-1 \in[-1,1], \quad i=1, \ldots, n ;
$$

see (41). Let us restate Lemmas 4.1 and 4.2 in terms of the $q_{i}$ 's: 
Lemma 4.4. Let $J \subset\{1, \ldots, n\}$ be a subset of cardinality $|J|=p \geqslant 1$. Then:

(a) For $2 p \leqslant i \leqslant n$ one has $q_{i} \leqslant 0$ with equality possible only for $i=2 p$.

(b) If $1 \leqslant i, j \leqslant 2 p$, then either $q_{i}=q_{j}$ or

$$
\left|q_{i}-q_{j}\right| \geqslant \frac{1}{2 p^{2}} .
$$

(c) If $q_{i}<0$ for some $1 \leqslant i \leqslant n$, then

$$
q_{i} \leqslant-\frac{1}{2 p+1} .
$$

(d) For $8 p^{3} \leqslant i \leqslant n$ one has

$$
-1 \leqslant q_{i} \leqslant-1+\frac{1}{4 p^{2}} .
$$

(e) A number distinct from -1 may appear in the sequence $q_{0}, \ldots, q_{n}$ at most $p$ times.

Next we consider the values $q_{i}^{\prime}=\phi_{J}\left(c_{i}^{\prime}\right)$ of the functional $\phi_{J}$ on the vertices of the simplex $B$ (see (19)).

Lemma 4.5. Let $J \subset\{1, \ldots, n\}$ be a subset of cardinality $|J|=p \geqslant 1$. Then:

(a) The numbers $q_{i}^{\prime}$ satisfy $-i \leqslant q_{i}^{\prime} \leqslant i$.

(b) For $2 p \leqslant i \leqslant n$ one has $q_{i}^{\prime} \leqslant 0$ with equality possible only for $i=2 p$.

(c) If $q_{i}^{\prime} \neq q_{j}^{\prime}$, then $\left|q_{i}^{\prime}-q_{j}^{\prime}\right| \geqslant 1$.

(d) If $q_{i}^{\prime}<0$ for some $1 \leqslant i \leqslant n$, then $q_{i}^{\prime} \leqslant-1$.

(e) A number may appear in the sequence $q_{0}^{\prime}, \ldots, q_{n}^{\prime}$ at most $p+1$ times.

Proof. Since $q_{i}^{\prime}=i q_{i}$, statements (a)-(d) follow from Lemma 4.4 and from the observation that $q_{i}^{\prime}$ is an integer. To prove $(e)$ we note that the numbers $q_{i}^{\prime}$ satisfy the following recurrence relation:

$$
q_{i+1}^{\prime}= \begin{cases}q_{i}^{\prime}-1 & \text { if } i+1 \notin J, \\ q_{i}^{\prime}+1 & \text { if } i+1 \in J .\end{cases}
$$

Hence the sequence $q_{0}^{\prime}, q_{1}^{\prime}, \ldots, q_{n}^{\prime}$ has exactly $p$ jumps up and decays between the jumps. This proves $(e)$.

Example 4.6. Consider the sequence $J=\{1,3, \ldots, 2 p-1\}$. Then

(i) $q_{i}^{\prime}=0$ for $i=0,2,4, \ldots, 2 p$,

(ii) $q_{i}^{\prime}=1$ for $i=1,3, \ldots 2 p-1$, and

(iii) $q_{i}^{\prime}<0$ for $i>2 p$.

We see that in this case the value zero appears in the sequence $q_{0}^{\prime}, \ldots, q_{n}^{\prime}$ exactly $p+1$ times. 


\section{Proof of Theorem 1.1 for $\mu=\mu_{a}$}

In this section we prove Theorem 1.1 for the measure $\mu=\mu_{a}$ described before the statement of Theorem 1.1.

Fix a subset $J \subset\{1,2, \ldots, n\}$ of cardinality $p \geqslant 1$ where we think of $p$ as being fixed and of $n$ as being large. Let $A$ be the simplex with vertices (18). Our first goal is to estimate the ratios of the form

$$
r_{J}=\frac{\operatorname{vol}\left(H_{J} \cap A\right)}{\operatorname{vol}(A)}
$$

where $H_{J} \subset \mathbf{R}^{n}$ is the half-space $H_{J}=\left\{v \in \mathbf{R}^{n} ; \phi_{J}(v)<0\right\}$. The average Betti numbers are sums of ratios of this kind; see Propositions 2.1 and 3.1. Recall that $\phi_{J}$ denotes the linear functional $\phi_{J}: \mathbf{R}^{n} \rightarrow \mathbf{R}$ given by (7).

The values of the functional $\phi_{J}$ on the vertices $c_{i}$ of $A$ are described in Lemma 4.4. In particular, by statement (a) of Lemma 4.4, for large $n$ the majority of the values $q_{i}$ are negative. More precisely, at most $2 p$ of them are positive. Hence, one may expect that the volume of the section $H_{J} \cap A$ approximates $\operatorname{vol}(A)$ for large $n$. This is indeed the case.

To estimate the difference $1-r_{J}$ from above we will apply Propositions 3.2 and 3.3 ; our aim is to show that it is exponentially small.

Proposition 5.1. Given an integer $p \geqslant 1$, there exist constants $C>0$ and $0<a<1$ such that for any $n \geqslant p$ and any subset $J \subset\{1,2, \ldots, n\}$ of cardinality $p$ one has

$$
1-r_{J}<C \cdot a^{n}
$$

It will be apparent from the proof that one can take for $a$ an arbitrary number satisfying $(2 p+1) /(2 p+2)<a<1$.

Proof. Consider the values $q_{i}=\phi_{J}\left(c_{i}\right)$, where $i=0,1, \ldots, n$. They may have multiplicities, i.e. the same value may appear several times. We will denote by $Q_{0}>Q_{1}>$ $, \ldots,>Q_{s} \in[-1,1]$ the different values of the sequence $q_{0}, \ldots, q_{n}$. Then there is a surjective mapping $\tau:\{0,1, \ldots, n\} \rightarrow\{0,1, \ldots, s\}$ such that $q_{i}=Q_{\tau(i)}$. For $0 \leqslant i \leqslant s$ we denote by $k_{i}+1$ the cardinality of the preimage $\tau^{-1}(i)$. Clearly,

$$
\sum_{i=0}^{s} k_{i}=n-s
$$

If $Q_{i} \leqslant 0$ for all $0 \leqslant i \leqslant s$ then $H_{J} \cap A=A$; hence $r_{J}=1$ and therefore (51) holds trivially. Thus without loss of generality we may assume that for some $0 \leqslant m \leqslant s$ one has $Q_{i}>0$ for $i=0, \ldots, m$ and $Q_{i} \leqslant 0$ for $i=m+1, \ldots, s$. By statement (a) of Lemma 4.4 we have

$$
\sum_{i=0}^{m}\left(k_{i}+1\right) \leqslant 2 p
$$

We also have

$$
k_{i} \leqslant p, \quad i=0,1, \ldots, s-1 ;
$$

see (e) of Lemma 4.4. Inequality (53) also applies to the multiplicity $k_{s}$ if $Q_{s}$ is distinct from -1 . 
Let $b=1+(2 p)^{-2}$. From statements $(a)$, (b), and (d) of Lemma 4.4 we obtain that for any $0 \leqslant i \leqslant m$ and $j \neq i$ one has

$$
\left|Q_{i}-Q_{j}\right| \geqslant \begin{cases}\left(2 p^{2}+1\right)^{-1}, & \text { for } 0 \leqslant j \leqslant 8 p^{3}, \\ \left(2 p^{2}\right)^{-1}-\left(-1+\frac{1}{4 p^{2}}\right)=b, & \text { for } 8 p^{3}<j \leqslant n .\end{cases}
$$

Let us explain the first line of this inequality. If $j$ satisfies $0 \leqslant j \leqslant 2 p$, then the statement follows from ( $a$ ) of Lemma 4.4. If $j>2 p$, then $Q_{j}<0$ and hence $Q_{i}-Q_{j} \geqslant$ $(2 p+1)^{-1} \geqslant\left(2 p^{2}+1\right)^{-1}$ by $(c)$ of Lemma 4.4 .

By applying Proposition 3.3 to $-\phi_{J}$ we have

$$
1-r_{J}=\sum_{i=0}^{m}\left[F_{i} \cdot \prod_{\substack{0 \leqslant j \leqslant s \\ j \neq i}}\left(\frac{Q_{i}}{Q_{i}-Q_{j}}\right)^{k_{j}+1}\right],
$$

where $F_{i}$ is given by

$$
\sum_{\delta \in P\left(s, k_{i}\right)}\left(\begin{array}{c}
n \\
\delta_{i}
\end{array}\right) \cdot\left(-Q_{i}\right)^{k_{i}-\delta_{i}} \cdot \prod_{\substack{0 \leqslant j \leqslant s \\
j \neq i}}\left(\begin{array}{c}
k_{j}+\delta_{j} \\
\delta_{j}
\end{array}\right) \cdot\left(Q_{i}-Q_{j}\right)^{-\delta_{j}} .
$$

We claim that for any $0 \leqslant i \leqslant m$,

$$
\left|F_{i}\right| \leqslant\left(2 p^{2}+1\right)^{p} n^{2 p}
$$

Indeed, observe that by (54),

$$
\left|Q_{i}-Q_{j}\right|^{-\delta_{j}} \leqslant\left(2 p^{2}+1\right)^{\delta_{j}}
$$

To estimate the binomial coefficient $\left(\begin{array}{c}k_{j}+\delta_{j} \\ \delta_{j}\end{array}\right)$ note that $\delta_{j} \leqslant k_{i}$ and thus $k_{j}+\delta_{j} \leqslant$ $k_{j}+k_{i} \leqslant n$; therefore

$$
\left(\begin{array}{c}
k_{j}+\delta_{j} \\
\delta_{j}
\end{array}\right) \leqslant n^{\delta_{j}} \quad \text { and } \quad\left(\begin{array}{c}
n \\
\delta_{i}
\end{array}\right) \leqslant n^{\delta_{i}}
$$

As $\left|Q_{i}\right| \leqslant 1$, each term in the sum (56) can be estimated as follows:

$$
\begin{aligned}
\left(\begin{array}{c}
n \\
\delta_{i}
\end{array}\right) \cdot\left(Q_{i}\right)^{k_{i}-\delta_{i}} \cdot \prod_{\substack{0 \leqslant j \leqslant s \\
j \neq i}}\left(\begin{array}{c}
k_{j}+\delta_{j} \\
\delta_{j}
\end{array}\right) \cdot\left(Q_{i}-Q_{j}\right)^{-\delta_{j}} \\
\leqslant\left(n \cdot\left(2 p^{2}+1\right)\right)^{\sum_{0}^{s} \delta_{j}} \leqslant\left(2 p^{2}+1\right)^{p} \cdot n^{p},
\end{aligned}
$$

where we have used that $\sum_{j=0}^{s} \delta_{j}=k_{i} \leqslant p$. The total number of terms in the sum (56) is

$$
\left|P\left(s, k_{i}\right)\right|=\left(\begin{array}{c}
s+k_{i} \\
k_{i}
\end{array}\right) \leqslant n^{p}
$$

since $s+k_{i} \leqslant n$ and $k_{i} \leqslant p$. This proves (57). 
Now consider the fractions $\frac{Q_{i}}{Q_{i}-Q_{j}}$ which appear in (55). For $0 \leqslant j \leqslant m, j \neq i$ we have by (54)

$$
\left|\frac{Q_{i}}{Q_{i}-Q_{j}}\right| \leqslant \frac{1}{\left|Q_{i}-Q_{j}\right|} \leqslant 2 p^{2}+1 \text {. }
$$

Note that $Q_{j}<Q_{m+1} \leqslant 0$ for any $m+2 \leqslant j \leqslant s$. Using Lemma 4.4 (c), we have for $m+2 \leqslant j \leqslant s$ and $1 \leqslant i \leqslant m$,

$$
0<\frac{Q_{i}}{Q_{i}-Q_{j}} \leqslant \frac{Q_{i}}{Q_{i}+(2 p+1)^{-1}} \leqslant \frac{1}{1+(2 p+1)^{-1}}=\frac{2 p+1}{2 p+2} .
$$

If $Q_{m+1}<0$, then estimate (61) continues to hold, whereas if $Q_{m+1}=0$ then the corresponding factor equals 1 . By Lemma 4.4 (e), using $k_{m+1}+1 \leqslant p$, we get for $1 \leqslant i \leqslant m$

$$
\prod_{m<j \leqslant s}\left|\frac{Q_{i}}{Q_{i}-Q_{j}}\right|^{k_{j}+1} \leqslant a_{0}^{\left(\sum_{j=m+1}^{s}\left(k_{j}+1\right)-p\right)} \leqslant C^{\prime} \cdot a_{0}^{n},
$$

where the constant $C^{\prime}$ depends on $p$ only. The number of summands in formula (55) equals $m+1$; by (52) it is bounded above by $2 p$.

Combining inequalities (57), (60) and (62) we obtain an estimate of the form $1-r_{J}<C n^{\gamma} a_{0}^{n}$ where the constants $C$ and $\gamma$ depend on $p$ but are independent of $n$. This clearly gives (51).

The following statement is equivalent to Proposition 5.1; we will need it in the proof of Theorem 1.1.

Proposition 5.2. Given an integer $p \geqslant 1$, there exist constants $C>0$ and $0<a<1$ such that for any $n \geqslant p$ and any subset $J \subset\{1,2, \ldots, n\}$ of cardinality $n-p$ one has

$$
r_{J}<C \cdot a^{n} .
$$

Proof. The claimed statement follows from Proposition 5.1 by observing that $r_{J}=$ $1-r_{\bar{J}}$ where $\bar{J}$ denotes the complement of $J$ in $\{1, \ldots, n\}$.

Proof of Theorem 1.1 for $\mu=\mu_{a}$. By Propositions 2.1 and 3.1 we have

$$
b_{p}(n, \mu)=\sum_{J} r_{J}
$$

where $J \subset\{1, \ldots, n\}$ runs over all subsets containing 1 and being of cardinality either $p+1$ or $n-2-p$. By Proposition 5.1 each $r_{J}$ with $|J|=p+1$ contributes to $b_{p}(n, \mu)$ a quantity exponentially close to 1 , and by Proposition 5.2 each term $r_{J}$ with $|J|=$ $n-2-p$ is exponentially small. Adding up all these contributions we arrive at the desired inequality (6).

\section{Proof of Theorem 1.1 for $\mu=\mu_{b}$}

The proof of Theorem 1.1 in the case $\mu=\mu_{b}$ is quite similar. Propositions 5.1 and 5.2 remain true but their proofs are slightly different. The difference between the 
two cases stems only from the different simplices involved: for $\mu=\mu_{a}$ we consider the simplex $A$ with vertices $c_{0}, \ldots, c_{n}$ and for $\mu=\mu_{b}$ we have to consider instead the simplex $B$ with vertices $c_{0}^{\prime}, \ldots, c_{n}^{\prime}$; see $(19)$.

Let us examine the arguments of the proof of Proposition 5.1 when $c_{i}$ is replaced by $c_{i}^{\prime}$. Inequality (52) follows from (b) of Lemma 4.5 and inequality (53) follows from statement (e) of Lemma 4.5. One introduces the points $Q_{0}^{\prime}>Q_{1}^{\prime}>\cdots>Q_{s}^{\prime}$ as the distinct values appearing in the sequence $q_{0}^{\prime}, q_{1}^{\prime}, \ldots, q_{n}^{\prime}$. Instead of (54) we have a simpler inequality $\left|Q_{i}^{\prime}-Q_{j}^{\prime}\right| \geqslant 1$, where $i \neq j$ which is a consequence of Lemma 4.5 (c).

Let us assume that $Q_{i}^{\prime}>0$ for $i=0, \ldots, m$ and $Q_{i}^{\prime} \leqslant 0$ for $i=m+1, \ldots, s$. We claim that for any $0 \leqslant i \leqslant m$ the quantity $F_{i}$ given by (56) satisfies inequality (57). Indeed, $\left|Q_{i}^{\prime}\right| \leqslant 2 p$ for $0 \leqslant i \leqslant m$ (see $(a)$ and (b) of Lemma 4.5), and hence

$$
\left|F_{i}\right| \leqslant \sum_{\delta \in P\left(s, k_{i}\right)}(2 p)^{k_{i}-\delta_{i}} \cdot n^{\sum \delta_{j}} \leqslant(2 p)^{p} \cdot n^{2 p} \leqslant\left(2 p^{2}+1\right)^{p} n^{2 p} .
$$

Here we have used inequalities (58) and (59). To estimate (55) from above we note that for $0 \leqslant i \leqslant m$ and $j \neq i$ one has

$$
\left|\frac{Q_{i}^{\prime}}{Q_{i}^{\prime}-Q_{j}^{\prime}}\right| \leqslant \begin{cases}\left|Q_{i}^{\prime}\right| \leqslant 2 p & \text { for } j \leqslant m \\ 1 & \text { for } j=m+1 \\ \frac{Q_{i}^{\prime}}{Q_{i}^{\prime}+1} \leqslant \frac{2 p}{2 p+1} & \text { for } j \geqslant m+2 .\end{cases}
$$

Here we have used statement (d) of Lemma 4.5. Combining the obtained inequalities we obtain that the statement analogous to Proposition 5.1 holds for $\mu=\mu_{b}$. The remaining arguments of the proof of Theorem 1.1 for $\mu=\mu_{b}$ are very similar to those described in the case $\mu=\mu_{a}$.

\section{References}

[1] M. Ali, Content of the frustrum of a simplex, Pacific J. Math. 48 (1973), 313-322.

[2] H.B. Curry and I.J. Schoenberg, On Pólya frequency functions IV: the fundamental spline functions and their limits, J. d'Analyse Math. 17 (1966), 71-107.

[3] M. Farber, Topology of random linkages, Algebr. Geom. Topol. 8 (2008), 155171.

[4] M. Farber and D. Schütz, Homology of planar polygon spaces, Geometria Dedicata 125 (2007), 75-92.

[5] L. Gerber, The volume cut off a simplex by a half-space, Pacific J. Math. 94 (1981), 311-314.

[6] J.-Cl. Hausmann and A. Knutson, The cohomology ring of polygon spaces, Ann. Inst. Fourier (Grenoble) 48 (1998), 281-321.

[7] M. Kapovich, and J. L. Millson, On the moduli space of polygons in the Euclidean plane, J. Differential Geom. 42 (1995), 430-464.

[8] D. G. Kendall, D. Barden, T.K. Carne, and H. Le, Shape and shape theory, John Wiley \& Sons Ltd., Chichester 1999. 
[9] A.A. Klyachko, Spatial polygons and stable configurations of points in the projective line, in Algebraic Geometry and its Applications, Aspects Math. E25, Vieweg, Braunschweig, 1994, 67-84.

[10] L.M. Milne-Thomson, The calculus of finite differences, MacMillan and Co. Ltd., London, 1933

[11] W. Thurston and J. Weeks, The mathematics of three-dimensional manifolds, Scientific American, July 1986, 94-106.

[12] G. Varsi, The multidimensional content of the frustrum of the simplex, Pacific J. Math. 46 (1973), 303-314.

[13] K. Walker, Configuration spaces of linkages, Undergraduate thesis, Princeton, 1985.

Michael Farber Michael.Farber@durham.ac.uk

Department of Mathematical Sciences, Durham University, Durham DH1 3LE, United Kingdom

Thomas Kappeler tk@math.unizh.ch

Institute of Mathematics, University of Zurich, 8057 Zurich, Switzerland 